\section{Vitalidade do recém-nascido por tipo de parto no Estado de São Paulo, Brasil}

\author{
Apgar score associated with mode of delivery in \\ São Paulo State, Brazil
}

\footnotetext{
${ }^{1}$ Laboratório de Economia Social, São Paulo, Brasil.

2 Secretaria de Economia e Planejamento do Estado de São Paulo, São Paulo, Brasil. 3 Hospital das Clínicas,

Faculdade de Medicina, Universidade de São Paulo, São Paulo, Brasil.

Correspondência S. Kilsztajn

Laboratório de Economia Social.

Rua Marquês de Paranaguá 164, apto. 602, São Paulo, SP 01303-050, Brasil.

skilmail@gmail.com
}

\section{Abstract}

This paper analyzes Apgar score associated with mode of delivery for live births in São Paulo State, Brazil, 2003. Based on data from the State Data Analysis System Foundation (SEADE), logistic regression was analyzed for live births with low Apgar score (0 to 6) at the fifth minute of life per mode of delivery, adjusted for obstetric, demographic, and social factors. Birth weight, gestational age at delivery, number of prenatal visits, maternal age, marital status, and years of schooling were statistically significant, and (as expected) all exposure categories were associated with low Apgar score. In the unadjusted model, the odds ratio (OR) for low Apgar was 0.890 (95\%CI: 0.836-0.948) for cesarean section compared to vaginal delivery. Nevertheless, in the adjusted model the OR was 1.045 (95\% CI: 0.977-1.117) for cesarean section compared to vaginal delivery. Adjusted for obstetric, demographic, and social factors, mode of delivery was not statistically significant for low Apgar.

Live Birth; Newborn Infant; Parturition; Apgar Score
Samuel Kilsztajn 1

Erika de Souza Lopes 1

Manuela Santos Nunes do Carmo 1,2

Ana Margarita de Andrade Reyes 3

\section{Introdução}

O Brasil apresenta a maior taxa de cesariana do mundo 1, e o percentual de partos cesáreos no Sistema Único de Saúde (SUS) e na rede privada do Estado de São Paulo atingiu, respectivamente, 32,9 e $80,4 \%$ em 2003 2. A cultura médica prócirúrgica prevalecente hoje no Brasil $3,4,5$ condiciona a avaliação clínica dos obstetras e atinge indistintamente tanto as cesáreas eletivas como as cesáreas de emergência indicadas, a princípio, para solucionar problemas decorrentes de complicações de partos vaginais.

A Organização Mundial da Saúde (OMS) considera que não há justificativa para um percentual de partos cesáreos superior a $15 \%$ em nenhuma região do mundo ${ }^{6}$. O Ministério da Saúde do Brasil, igualmente, considera que elevadas taxas de cesáreas são fatores determinantes da morbimortalidade materna e perinatal 7 .

Apesar do considerável avanço técnico nas práticas cirúrgicas, os resultados de diversas pesquisas confirmam que o parto cesáreo continua apresentando maior risco de morbimortalidade materna do que o parto vaginal, mesmo controladas a idade da mãe e a presença de hipertensão, outros transtornos, problemas e complicações 2,8,9. Os recentes trabalhos que concluíram que a morbimortalidade materna não está associada ao tipo de parto 10,11 foram conduzidos a partir de tamanhos de amostras que não viabilizam os modelos (testes t de Student e exato de 
Fisher 10; regressão logística múltipla 11) utilizados nas análises ${ }^{2}$. A morbimortalidade perinatal, por sua vez, pode estar associada ao parto cesáreo 12,13,14 ou, alternativamente, não estar relacionada ao tipo de parto 15,16,17,18.

$\mathrm{O}$ índice de Apgar, largamente utilizado para mensurar a vitalidade do recém-nascido, varia de 0 a 10 e avalia cinco sintomas objetivos: freqüência cardíaca (ausente: 0 ; < 100/min: 1; $>$ 100/min: 2); respiração (ausente: 0; fraca/irregular: 1; forte/choro: 2); irritabilidade reflexa (ausente: 0; algum movimento: 1; espirros/choro: 2); tônus muscular (flácido: 0; flexão de pernas e braços: 1; movimento ativo/boa flexão: 2) e cor (cianótico/pálido: 0; cianose de extremidades: 1; rosado: 2) 19. A importância do índice de Apgar como indicador de risco para a morbimortalidade neonatal tem sido ratificada em várias pesquisas recentes 15,20,21.

O objetivo deste trabalho é analisar a vitalidade do recém-nascido por tipo de parto para os nascidos vivos do Estado de São Paulo em 2003, controlados o peso da criança ao nascer, duração da gestação, número de consultas pré-natal, idade, estado civil e anos de estudo da mãe.

\section{Métodos}

Para a análise da vitalidade do recém-nascido por tipo de parto no Estado de São Paulo em 2003, foram utilizadas as informações das declarações de nascimento processadas pela Fundação Sistema Estadual de Análise de Dados (Fundação SEADE) 22 referentes aos campos índice de Apgar no quinto minuto e tipo de parto; às variáveis controle de peso da criança ao nascer, duração da gestação, número de consultas pré-natal, idade, estado civil e anos de estudo da mãe; ao campo "Detecta alguma malformação congênita elou anomalia cromossômica e tipo de gravidez (única ou múltipla)?". As variáveis ocupação habitual da mãe, filhos tidos nascidos vivos e tidos mortos não puderam ser utilizadas na análise em face do grande número de lacunas nesses campos da declaração de nascimento.

Foram excluídos da análise os nascidos vivos que apresentam risco extremo para baixa vitalidade e/ou indicação para parto cesáreo: nascidos vivos com peso menor que $1.500 \mathrm{~g}$ (peso muito baixo ao nascer) ou maior que 3.999g (macrossômicos); gestações com duração menor que 32 semanas ou maior que 41 semanas (pós-termo); nascidos vivos com malformação congênita e/ ou anomalia cromossômica detectada; gravidez múltipla.

Foram também excluídos da análise os nascidos vivos com declarações de nascimento com registro ignorado, incorreto ou sem preenchimento em um ou mais dos campos utilizados para a análise. A única exceção foi o campo "Detecta alguma malformação congênita elou anomalia cromossômica?", dado o procedimento incorreto, mas habitual, no seu preenchimento. Confunde-se "detecta" com "há", e utilizam-se indistintamente as alternativas "não", "ignorado" e sem preenchimento para os casos de malformação e/ou anomalia não detectada. Nesse caso, foram considerados, para efeito da análise, os registros "não" e "ignorado", bem como os campos sem preenchimento.

Nascidos vivos com índices de Apgar de 7 a 10 no quinto minuto de vida foram considerados normais; índices de 0 a 6 foram considerados baixos. Utilizaram-se como independentes as variáveis: tipo de parto (vaginal para categoria de referência; cesáreo para categoria de exposição); peso da criança ao nascer (2.500-3.999g para categoria de referência; 1.500-2.499g para categoria de exposição); duração da gestação (37-41 semanas para categoria de referência; 32-36 para categoria de exposição); número de consultas pré-natal; idade, estado civil e anos de estudo da mãe (12 e mais para categoria de referência; 0-3, 4-7 e 8-11 para categorias de exposição). As categorias de referência para número de consultas pré-natal, idade e situação conjugal maternas foram construídas a partir das classes dos respectivos campos da declaração de nascidos vivos que apresentaram, como resultado, taxas de nascidos vivos com baixo índice de Apgar iguais ou superiores à média verificada para o Estado de São Paulo (categorias de exposição: classes com taxas inferiores à média). Nascidos vivos com malformação congênita e/ou anomalia cromossômica detectada e gravidez múltipla, como indicado, foram excluídos da análise.

Numa primeira aproximação, foram calculadas as taxas de nascidos vivos com baixo índice de Apgar ( 0 a 6 ) no quinto minuto de vida por mil nascidos vivos, por tipo de parto, e demais variáveis de ordem obstétrica, demográfica e social, com a utilização de teste de qui-quadrado para as diferenças entre as proporções.

A partir de regressão logística simples e múltipla, com intervalo de confiança de 95\% (IC95\%), foram analisadas as odds ratio (OR) para baixa vitalidade do recém-nascido por tipo de parto e demais variáveis de ordem obstétrica, demográfica e social. Na regressão logística múltipla, foram controlados o peso da criança ao nascer, a duração da gestação, o número de consultas pré-natal, idade, estado civil e anos de estudo da mãe.

Para a análise estatística, foi utilizado o programa SPSS 13.0 (SPSS Inc., Chicago, Estados 
Unidos). As variáveis foram incluídas todas simultaneamente no modelo.

\section{Resultados}

A Fundação SEADE registrou um total de 610.630 nascidos vivos no Estado de São Paulo em 2003. Desse total, 8.035 apresentaram peso menor que 1.500g; 22.404, peso maior que 3.999g; 7.528, duração da gestação menor que 32 semanas; 4.032 duração da gestação maior que 41 semanas; 3.961 , malformação congênita e/ou anomalia cromossômica detectada; 12.145 gravidez múltipla. Os nascidos vivos que apresentaram risco extremo para baixa vitalidade e/ou indicação para parto cesáreo somaram, líquido de dupla contagem, 50.172 casos $(8,2 \%$ do total de nascidos vivos).

Dentre os 560.458 registros restantes, 64.713 declarações $(11,5 \%)$ não puderam ser utilizadas porque apresentavam pelo menos um dos campos mencionados em Métodos com registro ignorado, incorreto ou sem preenchimento (exceção feita à malformação congênita e/ou anomalia cromossômica detectada, conforme $M e^{-}$ todos). Dessa forma, puderam ser utilizados na análise de regressão logística 495.745 registros de nascidos vivos no Estado de São Paulo em 2003: 245.005 de partos vaginais e 250.740 de partos cesáreos. Estes últimos correspondem a uma taxa de 50,6\% (Tabela 1).

A taxa de nascidos vivos com índice de Apgar inferior a 7 registrou 7,9 por mil nascidos vivos no Estado de São Paulo em 2003; 8,4 para partos vaginais e 7,4 para partos cesáreos. Os resultados da pesquisa indicaram como referência para número de consultas pré-natal, idade e estado civil, respectivamente, as categorias sete e mais consultas pré-natal; mães de 21 a 35 anos de idade; casadas. Mães com menos de sete consultas pré-natal; com menos de 21 ou mais de 35 anos de idade e não casadas (separadas judicialmente,

Tabela 1

Vitalidade do recém-nascido por tipo de parto e outras variáveis. Estado de São Paulo, Brasil, 2003.

\begin{tabular}{|c|c|c|c|c|c|}
\hline \multirow[t]{2}{*}{ Variáveis } & \multicolumn{2}{|c|}{ Índice de Apgar no $5^{\circ}$ minuto } & \multirow{2}{*}{$\begin{array}{c}\text { Total } \\
(\mathrm{c}=\mathrm{a}+\mathrm{b})\end{array}$} & \multirow{2}{*}{$\begin{array}{c}(\mathrm{b} / \mathrm{c}) / \mathrm{mil} \\
\text { nascidos vivos }\end{array}$} & \multirow[t]{2}{*}{$\mathrm{p}$} \\
\hline & $7-10(a)$ & $0-6(b)$ & & & \\
\hline \multicolumn{6}{|l|}{ Tipo de parto * } \\
\hline Vaginal & 242.957 & 2.048 & 245.005 & 8,4 & \\
\hline Cesáreo & 248.872 & 1.868 & 250.740 & 7,7 & 0,000 \\
\hline \multicolumn{6}{|c|}{ Peso ao nascer (g) } \\
\hline $2.500-3.999$ & 458.190 & 3.099 & 461.289 & 6,7 & \\
\hline $1.500-2.499$ & 33.639 & 817 & 34.456 & 23,7 & 0,000 \\
\hline \multicolumn{6}{|c|}{ Duração da gestação (semanas) } \\
\hline $37-41$ & 465.838 & 3.177 & 469.015 & 6,8 & \\
\hline $32-36$ & 25.991 & 739 & 26.730 & 27,6 & 0,000 \\
\hline \multicolumn{6}{|c|}{ Número de consultas pré-natal } \\
\hline 7 e mais & 346.827 & 2.353 & 349.180 & 6,7 & \\
\hline $0-6$ & 145.002 & 1.563 & 146.565 & 10,7 & 0,000 \\
\hline \multicolumn{6}{|l|}{ Idade (anos) } \\
\hline $21-35$ & 336.975 & 2.494 & 339.469 & 7,3 & \\
\hline$<20 ;>35$ & 154.854 & 1.422 & 156.276 & 9,1 & 0,000 \\
\hline \multicolumn{6}{|l|}{ Estado civil } \\
\hline Casada & 211.409 & 1.362 & 212.771 & 6,4 & \\
\hline Não casada & 280.420 & 2.554 & 282.974 & 9,0 & 0,000 \\
\hline \multicolumn{6}{|l|}{ Anos de estudo } \\
\hline 12 e mais & 80.431 & 457 & 80.888 & 5,6 & \\
\hline $8-11$ & 224.330 & 1.707 & 226.037 & 7,6 & 0,011 \\
\hline $4-7$ & 152.908 & 13.363 & 154.271 & 8,8 & 0,000 \\
\hline $0-3$ & 34.160 & 389 & 34.549 & 11,3 & 0,000 \\
\hline Total & 491.829 & 3.916 & 495.745 & 7,9 & \\
\hline
\end{tabular}

Fonte: Fundação Sistema Estadual de Análise de Dados 22.

* Taxa de partos cesáreos: 50,6\% (250.740/495.745). 
união consensual, solteiras e viúvas) foram consideradas categorias de exposição (Tabela 1).

Tanto na regressão logística simples, quanto na regressão logística múltipla, o peso da criança ao nascer; duração da gestação; número de consultas pré-natal; idade, estado civil e anos de estudo da mãe resultaram ser todos significativos e, conforme esperado, todas as categorias de exposição apresentaram-se diretamente relacionadas à baixa vitalidade do recém-nascido. Ressalte-se, também, que, na regressão logística múltipla, todos os valores das OR diminuíram em relação aos valores registrados na regressão logística simples. A queda mais acentuada, como esperado, foi registrada nas variáveis peso e gestação, em virtude da forte correlação entre as categorias de exposição prematuridade e baixo peso ao nascer.

Na regressão logística simples para baixa vitalidade do recém-nascido, o parto cesáreo registrou OR de 0,890, com IC95\% entre 0,836 e 0,948 . Na regressão logística múltipla, entretanto, controlados o peso da criança ao nascer; duração da gestação; número de consultas pré-natal; idade, estado civil e anos de estudo da mãe, o parto cesáreo registrou OR de 1,045, com IC95\% entre 0,977 e 1,117 (Tabela 2).

\section{Discussão}

De acordo com a Rede Interagencial de Informações para a Saúde (RIPSA) 23, as estatísticas vitais do Estado de São Paulo podem ser consideradas de excelente qualidade. A cobertura do Sistema de Informações sobre Nascidos Vivos (SINASC) é igual ou superior a $90 \%$ e a do Sistema de In- formações sobre Mortalidade (SIM), igual ou superior a $80 \%$. É importante registrar que quase 90\% das declarações de nascimento do estado em 2003 apresentaram informações simultâneas para os campos índice de Apgar no quinto minuto; tipo de parto; peso da criança ao nascer; duração da gestação; número de consultas pré-natal; idade, estado civil e anos de estudo da mãe; tipo de gravidez (única ou múltipla).

No presente estudo, com base na regressão logística simples, o parto cesáreo poderia ser considerado como fator de proteção para baixa vitalidade do recém-nascido. Entretanto, na regressão logística múltipla, controlados os fatores de ordem obstétrica, demográfica e social disponíveis na declaração de nascimento, a variável tipo de parto deixou de ser estatisticamente significativa. Em face da baixa taxa de nascidos vivos com índice de Apgar inferior a sete, a OR para baixa vitalidade do recém-nascido por tipo de parto é uma boa aproximação e pode ser analisada como risco relativo de baixa vitalidade do recém-nascido por tipo de parto (o mesmo se aplica para a análise das variáveis de controle).

De acordo com a Introdução deste trabalho, a cultura médica pró-cirúrgica prevalecente hoje no Brasil 3,4,5 condiciona a avaliação clínica dos obstetras e atinge indistintamente tanto as cesáreas eletivas, como as de emergência. Para a análise dos resultados desta pesquisa, entretanto, deve-se considerar que parte das cesáreas eletivas é realmente clinicamente indicada e que parte das cesáreas de emergência é efetivamente realizada para solucionar problemas decorrentes de complicações de partos vaginais. Nos casos rigorosamente indicados, seja para cesáreas ele-

Regressão logística dos fatores de risco para baixa vitalidade do recém-nascido *. Estado de São Paulo, Brasil, 2003.

\begin{tabular}{|c|c|c|c|c|c|c|}
\hline \multirow[t]{2}{*}{ Variáveis } & \multicolumn{2}{|c|}{ Categorias } & \multicolumn{2}{|c|}{ Regressão simples } & \multicolumn{2}{|c|}{ Regressão múltipla } \\
\hline & Referência & Exposição & OR & IC95\% & OR & IC95\% \\
\hline Tipo de parto & Vaginal & Cesáreo & 0,890 & $0,836-0,948$ & 1,045 & $0,977-1,117$ \\
\hline Peso ao nascer (g) & $2.500-3.999$ & $1.500-2.499$ & 3,591 & $3,322-3,882$ & 2,163 & $1,967-2,379$ \\
\hline Duração da gestação (semanas) & $37-41$ & $32-36$ & 4,169 & $3,845-4,521$ & 2,640 & $2,391-2,915$ \\
\hline Número de consultas pré-natal & 7 e mais & $0-6$ & 1,589 & $1,490-1,694$ & 1,248 & $1,164-1,337$ \\
\hline Idade (anos) & $21-35$ & $<20 ;>35$ & 1,241 & $1,162-1,325$ & 1,107 & $1,035-1,184$ \\
\hline Estado civil & Casada & Não casada & 1,414 & $1,323-1,510$ & 1,231 & $1,148-1,321$ \\
\hline \multirow[t]{3}{*}{ Anos de estudo } & 12 e mais & $8-11$ & 1,339 & $1,207-1,485$ & 1,202 & $1,081-1,336$ \\
\hline & & $4-7$ & 1,569 & $1,411-1,745$ & 1,342 & $1,201-1,500$ \\
\hline & & $0-3$ & 2,004 & $1,750-2,296$ & 1,716 & $1,491-1,975$ \\
\hline
\end{tabular}

Fonte: Fundação Sistema Estadual de Análise de Dados 22.

* Índice de Apgar no $5^{\circ}$ minuto: 7-10 (normal), 0-6 (baixo). 
tivas, seja para as de emergência, o comprometimento da vitalidade do recém-nascido poderia não ser decorrente do parto cesáreo e sim da condição clínica que motivou a indicação; nesses casos, a cesárea pode mesmo contribuir para a sobrevivência fetal.

No presente trabalho esse viés foi atenuado pela exclusão, para efeito da análise, dos nascidos vivos que apresentaram risco extremo para baixa vitalidade e/ou indicação para parto cesáreo: nascidos vivos com peso menor que $1.500 \mathrm{~g}$ (peso muito baixo ao nascer) ou maior que $3.999 \mathrm{~g}$ (macrossômicos); gestações com duração menor que 32 semanas ou maior que 41 semanas (póstermo); nascidos vivos com malformação congênita e/ou anomalia cromossômica detectada; gravidez múltipla.

O índice de Apgar, como salientado, é um importante indicador de risco para a morbimortalidade perinatal. Estudo realizado para o $\mathrm{Mu}$ nicípio de São Paulo em 1998 considerou que o baixo índice de Apgar é um bom indicador de morbidade e está associado à mortalidade neonatal 15; outro estudo, desenvolvido para comparar condições sócio-econômicas e características neonatais no Rio de Janeiro em 1994, também concluiu que o índice de Apgar é um importante indicador de risco 20; finalmente, de acordo com estudo realizado no Hospital Universitário de Dakar entre 1994 e 2003, a mortalidade neonatal precoce atinge particularmente os nascidos vivos com baixo índice de Apgar 21.

Pesquisa realizada para nascidos vivos com peso igual ou maior que 2.500 g concluiu que a probabilidade de morte no período neonatal para parto cesáreo é 2,5 vezes maior do que a do parto vaginal e que este resultado não pode ser atribuído a características maternas ou fatores obstétricos 12. Estudo desenvolvido para partos cesáreos eletivos, partos cesáreos de emergência e partos vaginais concluiu que o risco de problemas respiratórios é menor para nascidos vivos de partos vaginais 13. De acordo com trabalho realizado para partos a termo, recém-nascidos de mulheres que se submeteram a parto cesáreo eletivo em gestações sem complicações apresentaram maior risco de desenvolverem problemas respiratórios comparados àqueles nascidos de partos vaginais 14 .

Em relação à mortalidade neonatal, pesquisa realizada para o Município de São Paulo em 1998 não encontrou associação entre mortalidade neonatal e cesarianas 15. Estudo realizado em hospitais localizados em Ribeirão Preto, no Estado de São Paulo, concluiu que o decréscimo da taxa de mortalidade perinatal não está associado ao crescimento da taxa de partos cesáreos 16 . Pesquisas realizadas em outros países, pobres 17 ou ricos 18 , concluíram que as crescentes taxas de cesariana não reduziram a taxa de mortalidade perinatal.

Considerando-se, por outro lado, que, apesar do avanço técnico nas práticas cirúrgicas, a morbimortalidade materna continua associada ao parto cesáreo 2,8,9, os partos cirúrgicos só deveriam ser empregados quando rigorosamente indicados. Contudo, no processo de medicalização da gestação, as cesáreas são hoje consideradas no Brasil a forma moderna e conveniente de parto ${ }^{3}$. Os médicos têm preferido os partos cesáreos porque são mais rápidos que os morosos partos vaginais 4 e utilizam os motivos manifestos por um pequeno número de mulheres que demanda partos cesáreos para generalizar a suposta preferência das gestantes pela cesariana 5 .

Os trabalhos reunidos na obra Humanizando Nascimentos e Partos 24 consideram que, no processo de medicalização da assistência ao parto, as gestações passaram a ser classificadas como de alto, médio e baixo risco - e, portanto, na atualidade, não há gestação sem risco. Além disso, a escola e a residência médica no Brasil não desenvolvem as habilidades necessárias para o trabalho de parto e estimulam o parto cesáreo, que passou a ser considerado o método "normal" de parto 24 . 


\section{Resumo}

Este trabalho analisa a vitalidade do recém-nascido por tipo de parto para os nascidos vivos do Estado de São Paulo, Brasil, em 2003. Com base nos dados das declarações de nascimento processadas pela Fundação Sistema Estadual de Análise de Dados (Fundação $S E A D E)$ e na regressão logística, foram analisadas as odds ratio (OR) para nascidos vivos com baixo índice de Apgar ( 0 a 6 ) no quinto minuto de vida por tipo de parto, controlados os fatores de ordem obstétrica, demográfica e social. Tanto na regressão simples, quanto na regressão múltipla, o peso da criança ao nascer; duração da gestação; número de consultas pré-natal; idade, estado civil e anos de estudo da mãe resultaram ser todos significativos e, conforme esperado, todas as categorias de exposição apresentaram-se diretamente relacionadas à baixa vitalidade do recém-nascido. $\mathrm{Na}$ regressão logística simples para baixa vitalidade do recém-nascido, o parto cesáreo, em relação ao parto vaginal, registrou OR de 0,890 (IC95\%: 0,836-0,948). Entretanto, na regressão logística múltipla, o parto cesáreo registrou OR de 1,045 (IC95\%: 0,977-1,117). Portanto, a variável tipo de parto não resultou ser estatisticamente significativa para baixa vitalidade do recém-nascido.

Nascido Vivo; Recém-Nascido; Parto; Índice de Apgar

\section{Referências}

1. International Cesarean Awareness Network. International cesarean and VBAC rates. Redondo Beach: International Cesarean Awareness Network; 2004

2. Kilsztajn S, Carmo MS, Machado Jr. LC, Lopes ES, Lima LZ. Caesarean sections and maternal mortality in São Paulo. Eur J Obstet Gynecol Reprod Biol 2007; 132:64-9.

3. Barbosa GP, Giffin K, Angulo-Tuesta A, Gama AS, Chor D, D'Orsi E, et al. Parto cesáreo: quem o deseja? Em quais circunstâncias? Cad Saúde Pública 2003; 19:1611-20.

4. Hopkins K. Are Brazilian women really choosing to deliver by caesarean? Soc Sci Med 2000; 51:725-40.

5. Faúndes A, Pádua KS, Osis MJD, Cecatti G, Souza MH. Opinião de mulheres e médicos brasileiros sobre a preferência pela via de parto. Rev Saúde Pública 2004; 38:488-94.

6. Appropriate technology for birth. Lancet 1985; 326:436-7.

\section{Colaboradores}

Todos os autores participaram de todas as etapas da pesquisa, contribuindo no levantamento bibliográfico, com os dados estatísticos, nas análises estatísticas e na redação.

\section{Agradecimentos}

A Luis Carlos Machado Jr., médico obstetra do Centro de Saúde Escola, Faculdade de Medicina, Universidade de São Paulo e da Faculdade de Medicina do ABC, e a Daphne Rattner, da Área Técnica de Saúde da Mulher do Ministério da Saúde, pela colaboração; aos pareceristas dos Cadernos de Saúde Pública, pelas sugestões.
7. Ministério da Saúde. Parto, aborto e puerpério, assistência humanizada à mulher. Brasília: Ministério da Saúde; 2001.

8. Francome C, Savage W. Caesarean section in Britain and the United States $12 \%$ or $24 \%$ : is either the right rate? Soc Sci Med 1993; 37:1199-218.

9. Harper MA, Byington RP, Espeland MA, Naughton M, Meyer R, Lane K. Pregnancy-related death and health care services. Obstet Gynecol 2003; 102:2738.

10. Nomura RMY, Alves EA, Zugaib M. Complicações maternas associadas ao tipo de parto em hospital universitário. Rev Saúde Pública 2004; 38:9-15.

11. Lydon-Rochelle M, Holt VL, Easterling TR, Martin DP. Cesarean delivery and postpartum mortality among primiparas in Washington State, 1987-1996. Obstet Gynecol 2001; 97:169-74. 
12. Bobadilla JL, Walker GJA. Early neonatal mortality and cesarean delivery in Mexico City. Am J Obstet Gynecol 1991; 164:22-8.

13. Levine EM, Ghai V, Barton JJ, Strom CM. Mode of delivery and risk of respiratory diseases in newborns. Obstet Gynecol 2001; 97:439-42.

14. Zanardo V, Simbi AK, Franzoi M, Solda G, Salvadori A, Trevisanuto D. Neonatal respiratory morbidity risk and mode of delivery at term: influence of timing of elective caesarean delivery. Acta Paediatr 2004; 93:643-7.

15. Machado CJ, Hill K. Maternal, neonatal and community factors influencing neonatal mortality in Brazil. J Biosoc Sci 2005; 37:193-208.

16. Duarte G, Coltro PS, Bedone RV, Nogueira AA, Gelonezzi GM, Franco LJ. Trends in the modes of delivery and their impact on perinatal mortality rates. Rev Saúde Pública 2004; 38:379-84.

17. Mukherjee J, Bhattacharya PK, Lahiri TK, Samaddar JC, Mehta R. Perinatal mortality in caesarean section: a disturbing picture of unfulfilled expectations. J Indian Med Assoc 1993; 91:202-3.

18. Jonsdottir G, Bjarnadottir RI, Geirsson RT, Smarason A. No correlation between rates of caesarean section and perinatal mortality in Iceland. Laeknabladid 2006; 92:191-5.
19. Apgar V. A proposal for a new method of evaluation of the newborn infant. Curr Res Anesth Analg 1953; 32:260-7.

20. D'Orsi E, Carvalho MS, Cruz OG. Similarity between neonatal profile and socioeconomic index: a spatial approach. Cad Saúde Pública 2005; 21:786-94.

21. Cisse CT, Yacoubou Y, Ndiaye O, Diop-Mbengue $\mathrm{R}$, Moreau JC. Time-course of neonatal precocious mortality between 1994 and 2003 at the Dakar University Teaching Hospital. J Gynecol Obstet Biol Reprod (Paris) 2006; 35:46-52.

22. Fundação Sistema Estadual de Análise de Dados. Estatísticas vitais paulistas: banco de dados nascidos vivos 2003 [CD-ROM]. São Paulo: Fundação Sistema Estadual de Análise de Dados; 2004.

23. Rede Interagencial de Informações para a Saúde. Indicadores e dados básicos - Brasil, 2004. Brasília: Ministério da Saúde; 2006.

24. Rattner D, Trench B, organizadores. Humanizando nascimentos e partos. São Paulo: Editora Senac; 2005 .

Recebido em 25/Jul/2006

Versão final reapresentada em 07/Dez/2006 Aprovado em 09/Fev/2007 